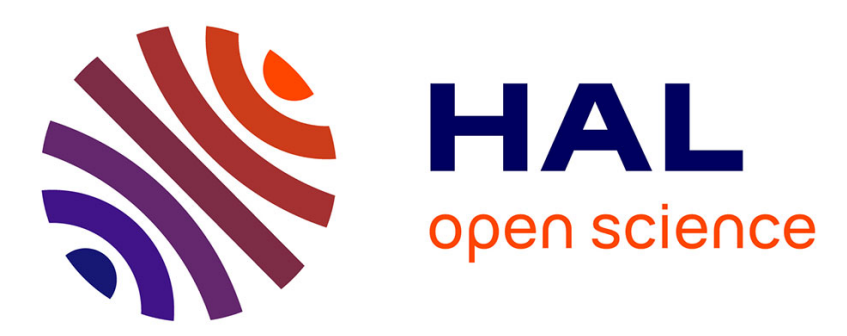

\title{
Jaugeage par radar mobile, protocole et résultats
}

Guillaume Dramais, J. Le Coz, R. Le Boursicaud, A. Hauet, M. Lagouy

\section{To cite this version:}

Guillaume Dramais, J. Le Coz, R. Le Boursicaud, A. Hauet, M. Lagouy. Jaugeage par radar mobile, protocole et résultats. La Houille Blanche - Revue internationale de l'eau, 2014, 3, pp.23-29. 10.1051/lhb/2014025 . hal-01118426

\section{HAL Id: hal-01118426 https://hal.science/hal-01118426}

Submitted on 19 Feb 2015

HAL is a multi-disciplinary open access archive for the deposit and dissemination of scientific research documents, whether they are published or not. The documents may come from teaching and research institutions in France or abroad, or from public or private research centers.
L'archive ouverte pluridisciplinaire HAL, est destinée au dépôt et à la diffusion de documents scientifiques de niveau recherche, publiés ou non, émanant des établissements d'enseignement et de recherche français ou étrangers, des laboratoires publics ou privés. 


\title{
Jaugeage par radar mobile, protocole et résultats
}

\author{
Guillaume DRAMAIS ${ }^{1}$, Jérôme LE COZ¹, Raphaël LE BOURSICAUD ${ }^{1}$, Alexandre HAUET², Mickaël LAGOUY
}

1. Irstea - UR HH - 5 rue de la Doua,69626Villeurbanne,France-guillaume.dramais@irstea.fr

2.EDF-DTG-DMM - 21 avenue de l'Europe, 38000 Grenoble, France-alexandre.hauet@edf.fr

\begin{abstract}
RÉSUMÉ. - La technique de mesure des vitesses de surface par radar mobile séduit les équipes d'hydrométrie du fait de sa rapidité et de la simplicité de sa mise en œuvre pour des jaugeages en crue. Les radars mobiles viennent ainsi compléter la flotte d'instruments déjà disponible dans les équipes. L'intérêt de ces radars et de cette technique est de pouvoir explorer le champ de vitesse de surface sans contact avec la rivière. Le calcul du débit à partir de ces mesures partielles nécessite des mesures complémentaires, notamment de bathymétrie et de hauteur d'eau. L'évaluation du coefficient de vitesse est aussi un point important. Cette technique est particulièrement bien adaptée aux mesures en crue. Cette étude présente le protocole de mesure proposé par Irstea pour le jaugeage par radar mobile, qui propose notamment une solution de déploiement pour les cas où la hauteur d'eau varie très rapidement dans le cours d'eau. Des cas d'application sur des sites variés sont présentés notamment lorsque la technique par radar a été testée et comparée avec d'autres méthodes. Les résultats sont très encourageants.
\end{abstract}

Mots-clés : hydrométrie, mesure de vitesse, radar, crue, rivière

\section{Mobile radar gauging, field procedure and results}

\begin{abstract}
The non-contact surface velocity measurement using SVR (Surface Velocity Radar) can be used to measure river discharge during floods. This method convinces hydrometry services because it is quick and simple to apply. The SVR is a complimentary instrument to add to the field panoply, especially for flood measurements. For computing discharge from surface velocities, additional measurements (bathymetry, velocity profiles, water level) are needed, and a velocity coefficient has to be estimated for each site. Mean velocity and surface velocity are linked through this coefficient. This study explains the field procedure suggested by Irstea (National Research Institute of Science and Technology for Environment and Agriculture) for gauging streams with a SVR system, with a two-way procedure to improve the discharge measurement accuracy during fast flood with fast water level variations. Some application results are presented in different rivers, with a comparison between the radar method and other conventional discharge measurements. The quality of the results is very encouraging.
\end{abstract}

Key-words: hydrometry, velocity measurement, radar, flood, river

\section{INTRODUCTION}

Alors qu'elle est cruciale pour la qualité des enregistrements hydrologiques, la mesure du débit des crues est toujours délicate, car les conditions météorologiques sont la plupart du temps défavorables, les accès difficiles, le matériel mis à mal, les délais d'intervention très courts. Les mesures sans contact et en particulier la mesure de débit par radar mobile offrent plusieurs avantages :

- L'exposition limitée du personnel et du matériel à des conditions d'écoulement dangereuses,

- La facilité de déploiement du matériel,

- La rapidité de la mesure qui permet en conditions de crue de jauger rapidement un écoulement transitoire, et sur un bassin versant d'effectuer plusieurs jaugeages dans un secteur donné,

- Le faible coût du matériel.

Dans cette étude nous présenterons le principe du capteur et de la méthode. Dans un deuxième temps nous détaillerons les recommandations et le protocole proposé pour effectuer les mesures. Enfin nous présentons quelques résultats sur des cours d'eau variés où le jaugeage par radar mobile a pu être confronté à des techniques traditionnelles.

\section{LA TECHNIQUE DE JAUGEAGE PAR RADAR MOBILE}

\section{II.1. La mesure de vitesse}

Le principe de mesure du capteur est d'émettre des impulsions (ondes électromagnétiques) très courtes, et d'interpréter ensuite le signal retour. La plupart de ces capteurs émettent dans les bandes de fréquence ISM (Industriel, Scientifique, et Médical). Le modèle de radar testé dans cette étude est le capteur SVR (Decatur Electronics) utilisant la technologie FMCW (Frequency Modulated Continuous Waves) de fréquence $24.1 \mathrm{GHz}$. Les ondes émises par le radar sont réfléchies par la rugosité de la surface de l'écoulement (figure1), la fréquence rétrodiffusée étant alors modifiée proportionnellement à la vitesse de l'écoulement projetée sur l'axe de visée, selon un décalage Doppler. Il est donc nécessaire de connaitre l'angle d'inclinaison du radar sous l'horizontale (équation 1). Dans le cas du SVR Decatur, cet angle est fourni par un inclinomètre interne. Pour mesurer correctement la vitesse de l'écoulement le niveau de signal reçu doit donc être suffisant. Pour cela la longueur d'onde des vaguelettes résonantes doit respecter la condition de Bragg [Costa, 


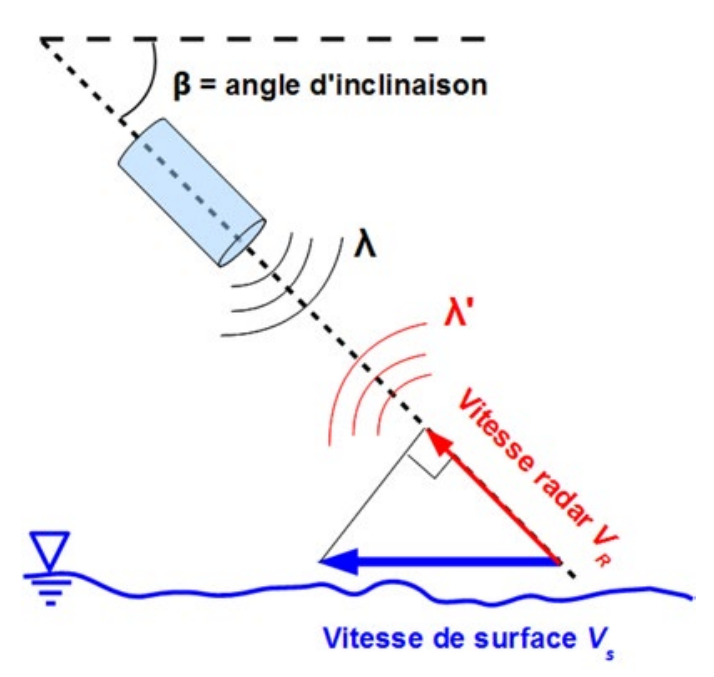

Fig. 1 : Mesure de vitesse : schéma de principe

2006]. En pratique, la technique fonctionne sur une surface d'écoulement suffisamment rugueuse (vaguelettes en surface d'une longueur d'onde de $0,9 \mathrm{~cm}$ pour le capteur SVR avec un angle incident de $45^{\circ}$ ). Augmenter l'angle de visée (compris entre $30^{\circ}$ et $60^{\circ}$ pour un SVR) permet d'augmenter le retour radar, mais augmente aussi les erreurs de projection de la vitesse.

\section{II.2. La mesure de la section mouillée}

Une mesure de bathymétrie doit être effectuée au droit du champ de vitesse exploré au radar, idéalement au moment de la mesure des vitesses, ce qui n'est pas toujours possible. Dans ce cas, on utilisera une bathymétrie réalisée avant et/ou après la crue, sous hypothèse que la section n'était pas sensiblement différente au moment du jaugeage. Cette mesure de bathymétrie peut être réalisée de différentes manières. Dans les petits cours d'eau on privilégiera les mesures de topographie optique par théodolite, tachéomètre ou niveau optique. Dans les grands cours d'eau l'utilisation d'un sondeur mono ou multifaisceau ou le suivi de fond d'un profileur de courant ADCP peuvent être utilisés. La longueur dévidée d'un câble de camion jaugeur ou tout autre système de mesure permettant des mesures de profondeur peuvent également être utilisés. L'aire de la section mouillée est ensuite calculée à partir de la bathymétrie et de la cote de l'eau au moment du jaugeage : les deux mesures doivent donc être référencées dans le même système, ou par rapport à un repère commun.

\section{II.3. Calcul du débit}

Les mesures radar permettent la mesure partielle du champ de vitesse, à la surface de l'écoulement uniquement. Pour

$$
V_{s}=\frac{V_{R}}{\cos \beta}
$$

Avec' $V_{s}$ la vitesse de surface

$V_{R}$ la vitesse mesurée par le radar en $\mathrm{m} / \mathrm{s}$ $\beta$ l'angle incident mesuré par le SVR en ${ }^{\circ}$ calculer un débit, on doit déterminer une vitesse moyenne dans la section. Pour cela on utilise généralement un modèle de distribution verticale des vitesses selon une loi logarithmique ou puissance. Le rapport entre la vitesse moyenne et la vitesse de surface est nommé " coefficient de vitesse » et noté $\alpha$ :

$$
V_{m}=V_{s} \times \alpha
$$

Avec $V_{m}$ la vitesse moyenne sur la verticale

$V_{s}$ la vitesse de surface

$\alpha$ le coefficient de vitesse

Ce coefficient de vitesse $\alpha$ est généralement évalué selon la norme ISO 748 [ISO, 2009] entre 0,80 pour les rivières peu profondes à fond rugueux et 0,91 pour des chenaux profonds à fond plus lisse, avec une valeur de 0,86 en conditions moyennes (cf. Tableau 1). La valeur peut aussi être déterminée expérimentalement à partir de mesures de profils verticaux de vitesse ou de jaugeages disponibles au site de mesure [Dramais 2011], [Hauet, 2008], [Le Coz, 2008, 2010]. Le calcul du débit est ensuite effectué conformément aux préconisations de la norme ISO 748 [ISO, 2009], par exemple avec le logiciel BAREME en plaçant la vitesse radar à la surface et en donnant simplement la valeur du coefficient de vitesse au coefficient de fond.

\section{II.4. Tests des capteurs}

La comparaison inter-laboratoire sur les mesures de débit organisée en septembre 2012 sur le Rhône à Génissiat par le Groupe Doppler [Le Coz, 2007] a permis de tester un lot de 10 radars SVR (Figure 2) simultanément à des mesures

Tableau 1 : Valeurs repères pour le coefficient de vitesse $\alpha$

\begin{tabular}{|l|c|}
\hline Valeur par défaut (écoulement uniforme, rugosité moyenne) & 0,86 \\
\hline Valeur extrême basse : écoulement non uniforme, torrents & 0,60 \\
\hline Ecoulement rugueux : faibles hauteurs d'eau, fond rugueux, rivière de piémont & 0,80 \\
\hline Valeurs usuelles & $0,84-0,88$ \\
\hline Ecoulement lisse : fortes hauteurs d'eau, fond lisse, canaux bétons, grands fleuves & 0,91 \\
\hline Valeur extrême haute : écoulement non uniforme, influence d'un ouvrage & 1,20 \\
\hline
\end{tabular}


par ADCP (Acoustic Doppler Current Profiler) et à d'autres modèles de radars vélocimétriques. Ces essais sont en cours d'analyse et feront l'objet d'une autre étude, mais deux points principaux émergent de cette campagne de mesure, confirmant les observations antérieures.

\section{II.4.1. L'angle d'inclinaison}

Les appareils SVR sont équipés d'un inclinomètre pour mesurer l'angle d'inclinaison du radar par rapport à la surface de l'eau, supposée horizontale. La qualité de ces mesures d'angle a été largement remise en question par différents travaux ([Zolezzi, 2011], [Esteves, 2012]). Lors de la comparaison inter-laboratoire, les inclinomètres ont été testés et des corrections d'angle ont été jugées nécessaires pour la plupart d'entre eux : la tendance était à la sous-estimation de l'angle et donc à la surestimation des vitesses, de l'ordre de $+10 \%$ en moyenne. Un retour collectif vers le constructeur a été réalisé, sans réponse satisfaisante pour l'instant. Ceci étant, ce défaut ne remet pas en cause l'intérêt pratique important de ce type d'instruments, car l'erreur d'angle peut aisément être corrigée en post-traitement par un ajustement du coefficient de vitesse. En cas de mesure contradictoire de l'angle d'inclinaison (ce qu'il est donc recommandé de faire pour chaque instrument et chaque angle de visée utilisé), la vitesse peut être corrigée en utilisant la formule 3 :

$$
V_{c}=\frac{V_{S} \times \cos \beta}{\cos \varphi}
$$

Avec $V_{c}$ la vitesse corrigée en $\mathrm{m} / \mathrm{s}$

$V_{S}$ la vitesse mesurée en $\mathrm{m} / \mathrm{s}$

$\beta$ l'angle incident mesuré par le SVR en ${ }^{\circ}$

$\varphi$ l'angle réel mesuré par un dispositif de contrôle d'angle en ${ }^{\circ}$

\section{II.4.2. L'intensité du signal}

Les ondes émises par le radar sont réfléchies par la rugosité de la surface de l'écoulement (voir II.1). Des études ont

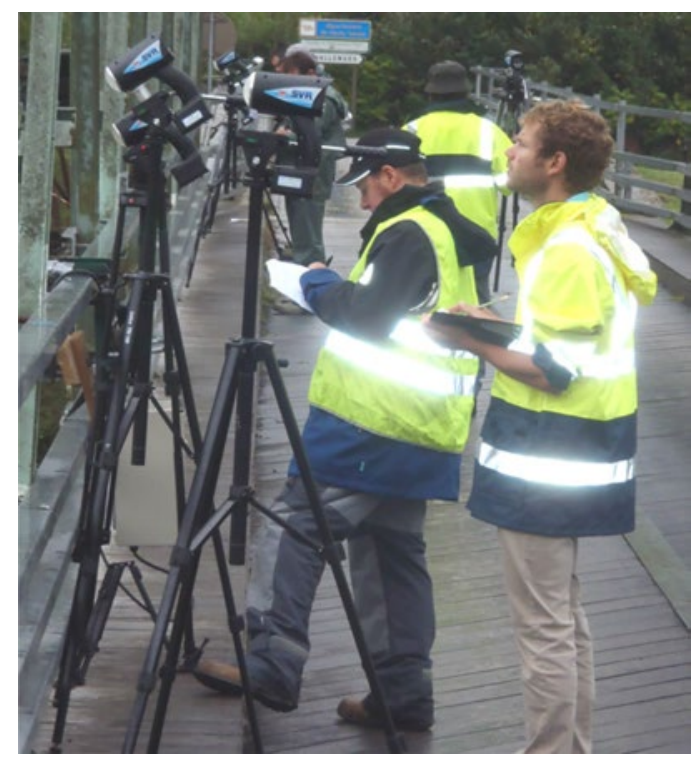

Fig. 2 : Comparaison sur le Rhône à Génissiat les données d'angles et de vitesses sont saisies sur des fiches de terrain. vérifié que pour des radars FMCW de type Kalesto v (Ott) le gain du signal rétrodiffusé devait être supérieur à $50 \mathrm{~dB}$ pour que la mesure de vitesse soit valable [Dramais, 2011]. Une surface trop lisse ne permet pas un bon retour du signal. Il a été observé des dispersions très importantes en vitesse dans des cas d'écoulement peu rugueux. La dispersion des 10 mesures de vitesses successives affichées par le SVR semble être un critère de qualité de la mesure finale. Sur les données de la comparaison inter-laboratoire à Génissiat en 2012, des dispersions importantes ont été observées jusqu'à $30 \%$ de variations de vitesse pour une série de mesures sur le même point avec le même appareil. Il est donc important de bien noter l'ensemble des 10 mesures instantanées de chaque tir, et il semble possible dans certains cas de pouvoir établir une moyenne correcte en éliminant les mesures aberrantes. A noter qu'il a été possible d'améliorer le retour radar en augmentant l'angle de visée, mais que cela augmente les erreurs d'angle de projection.

\section{PROTOCOLE DE TERRAIN}

\section{III.1. Avant le jaugeage}

Comme pour la plupart des jaugeages par exploration du champ des vitesses, il est nécessaire de choisir l'emplacement de mesure judicieusement. La section de mesure sera choisie rectiligne et présentant un écoulement homogène [ISO, 2009]. Le radar sera de préférence installé sur un trépied perpendiculairement à la section de jaugeage (figures $2 \& 3$ ). Le capteur peut pointer vers l'amont ou l'aval, sachant que l'aval des ponts présente souvent un écoulement moins homogène que l'amont (piles, modifications de largeur). L'angle d'inclinaison est calé le plus souvent à $45^{\circ}$. Il est préférable de garder le même angle d'inclinaison pendant le jaugeage, mais il peut être modifié si nécessaire pour éviter des obstacles, les bords, ou de la végétation, ou encore pour augmenter le retour radar et la répétabilité des vitesses. Il faut alors l'ajuster en restant entre $30^{\circ}$ et $60^{\circ}$.

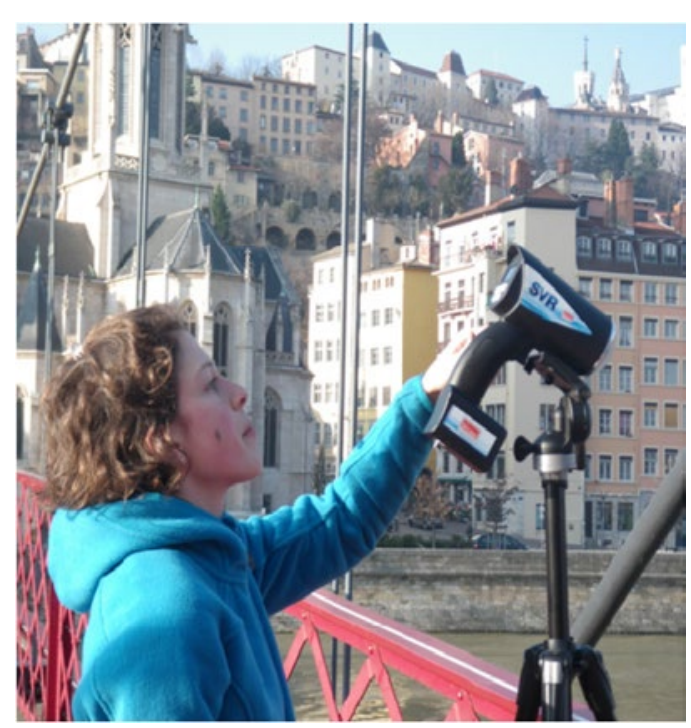

Fig. 3 : Le SVR sur un trépied, le capteur est dirigé vers la surface de l'écoulement, l'opératrice déclenche la mesure (Saône à St Georges). 


\section{III.2. Répartition des mesures}

L'originalité du protocole est de proposer de réaliser une première série de mesures sur le transect puis d'intercaler entre chaque position de nouveaux points lors d'un second passage. Par défaut, on mesurera les vitesses sur 13 points de mesure seulement, ce qui paraît un optimum pour conserver son caractère rapide et allégé à cette technique de jaugeage des crues. La largeur totale du cours d'eau est divisée par 7 pour placer 6 points sur un aller, puis 7 autres sont intercalés entre les 6 premiers sur le retour (figure 4). Ainsi, en cas de variation rapide du niveau (crues éclair par exemple), on pourra dépouiller séparément les deux jaugeages aller et retour. La position et le nombre de points de mesure de surface peuvent être adaptés pour mieux décrire l'écoulement principal, les ruptures de pente ou de vitesse, ou encore pour raccourcir la durée de jaugeage, en conservant toutefois un minimum de 10 positions au total. La mesure sur une position prend habituellement $2 \mathrm{~min}$, déplacement et manipulation compris, ce qui maintient la durée totale du jaugeage inférieure à 20-30min.

\section{III.3. Sensibilité de la mesure}

Les conditions météorologiques peuvent avoir une influence sur la mesure de vitesse par radar : le vent peut largement modifier la rugosité et la vitesse apparente de la surface d'un cours d'eau. Une pluie battante pourrait également avoir une influence non négligeable, quoique nous n'ayons jamais pu le mettre en évidence formellement. L'influence de ces deux phénomènes est difficile à évaluer. Le moins bon résultat de mesure de débit présenté dans cette étude (La Bourbre à Jameyzieu), avec $+13 \%$ d'écart à la valeur dite de référence (tableau 2), a été obtenu en conditions pluvieuses, mais le lien n'est pas démontré. Dans des cas susceptibles d'être défavorables il est nécessaire de répéter les mesures pour déceler d'éventuelles dispersions fortes sur la mesure de vitesse qui pourraient inciter à préférer une autre méthode de jaugeage, ou bien ré-évaluer à la hausse l'incertitude de mesure.

\section{III.4. Mesures complémentaires}

L'élément essentiel pour que la bathymétrie de la section de mesure (cf. II.2) soit exploitable est qu'elle soit dans le même référentiel topographique que l'échelle qui permettra de contrôler les variations de hauteur d'eau. La mesure de hauteur d'eau lue à l'échelle avant et après la mesure est indispensable, en cas de crue rapide le contrôle de hauteur d'eau peut être fait quasiment à chaque position de mesure. Ces mesures de hauteur d'eau permettent d'affiner le calcul de la section mouillée.

En l'absence d'échelle limnimétrique, il faut raccorder le niveau d'eau à la géométrie de la section (par exemple en mesurant le tirant d'air sous le pont, avec pour origine un repère fixe à relier plus tard à la topographie de la section).

Il est important de bien documenter les conditions du jaugeage : emplacement précis de la visée, repère des abscisses et des cotes, variations du niveau, conditions météorologiques, aspect de l'écoulement (régularité, uniformité, proximité d'ouvrages, d'obstacles ou de singularités, rugosité de la surface, nature du fond, etc.), et de prendre des photos ou des films de l'écoulement. Ces informations permettront d'affiner et de justifier la valeur et l'incertitude estimées du coefficient de vitesse, de la bathymétrie, et finalement du débit.

\section{RESULTATS DE JAUGEAGE}

Les radars mobiles SVR ont été déployés depuis 2011 dans le cadre de différents suivis de cours d'eau dans la région Rhône-Alpes. Les principaux résultats de comparaisons à des débits de référence sont présentés dans le tableau 2, par ordre croissant de débit. Ces jaugeages ont été dépouillés avec le logiciel BAREME [Bechon, 2012] de la DREAL Rhône Alpes qui permet des dépouillements avec un point unique de vitesse en surface et la possibilité d'ajuster le coefficient de vitesse (identique coefficient de fond). Ces comparaisons des jaugeages radar avec d'autres techniques d'hydrométrie couvrent une gamme de conditions étendue, plus que les essais disponibles dans la littérature, par exemple par [Fulton, 2008]. Sur les petits cours d'eau, les jaugeages radar sont comparés à des jaugeages par exploration du champ des vitesses à l'aide d'un ADV (Acoustic Doppler Velocimeter) sur perche à pied. Sur les cours d'eau plus importants les mesures ont été comparées à des jaugeages ADCP, LSPIV (Large Scale Particle Image Velocimetry), ou encore au moulinet associé à un camion

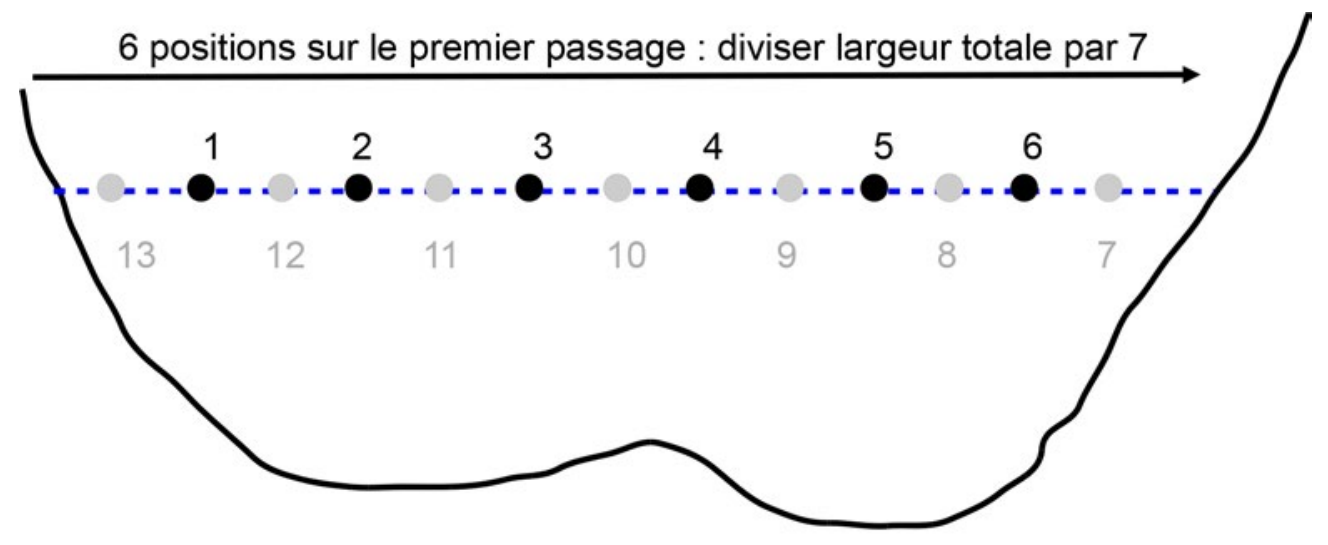

7 positions sur traversée retour : intercaler les points de mesure

Figure 4 : Répartition des points de mesures sur la section. 
Tableau 2 : Résultats des comparaisons entre radar et mesures classiques.

\begin{tabular}{|c|c|c|c|c|c|c|}
\hline Site & $\begin{array}{c}\text { Débit radar } \\
{\left[\mathrm{m}^{3} / \mathbf{s}\right]} \\
\text { (modèle) }\end{array}$ & $\begin{array}{c}\text { Méthode } \\
\text { de référence }\end{array}$ & $\begin{array}{c}\text { Débit(s) } \\
\text { de référence } \\
{\left[\mathbf{m}^{3} / \mathbf{s}\right]}\end{array}$ & Ecart(s) & $\begin{array}{c}\text { Technique } \\
\text { de mesure } \\
\text { de bathymétrie }\end{array}$ & $\begin{array}{l}\text { Coefficient } \\
\text { de vitesse }\end{array}$ \\
\hline $\begin{array}{l}\text { Le Charbonnières } \\
\text { à Charbonnières-les-bains } \\
(69, \text { Irstea) }\end{array}$ & $\begin{array}{l}0,885 \\
(\mathrm{SVR})\end{array}$ & $\begin{array}{c}\text { ADV } \\
\text { courbe } \\
\text { de tarage }\end{array}$ & $\begin{array}{l}0,881 \\
0,825\end{array}$ & $\begin{array}{l}+0,4 \% \\
+7,3 \%\end{array}$ & Perche graduée & $\begin{array}{l}0,86 \text { valeur } \\
\text { par défaut }\end{array}$ \\
\hline $\begin{array}{l}\text { Le Ratier à } \\
\text { Saint-Genis-les-Ollières } \\
(69, \text { Irstea) }\end{array}$ & $\begin{array}{c}1,00 \\
(\mathrm{SVR})\end{array}$ & $\begin{array}{c}\text { courbe } \\
\text { de tarage }\end{array}$ & 0,877 & $+14 \%$ & $\begin{array}{c}\text { Mesure } \\
\text { topographique } \\
\text { (station totale) }\end{array}$ & $\begin{array}{l}0,86 \text { valeur } \\
\text { par défaut }\end{array}$ \\
\hline $\begin{array}{l}\text { Le Ratier à } \\
\text { Saint-Genis-les-Ollières } \\
(69, \text { Irstea) }\end{array}$ & $\begin{array}{c}1,09 \\
(\mathrm{SVR})\end{array}$ & $\begin{array}{c}\text { ADV } \\
\text { courbe } \\
\text { de tarage }\end{array}$ & $\begin{array}{l}1,05 \\
1,11\end{array}$ & $\begin{array}{l}+3,8 \% \\
-1,8 \%\end{array}$ & Perche graduée & $\begin{array}{l}0,86 \text { valeur } \\
\text { par défaut }\end{array}$ \\
\hline $\begin{array}{l}\text { Le Ratier à } \\
\text { Saint-Genis-les-Ollières } \\
(69, \text { Irstea) }\end{array}$ & $\begin{array}{c}1,29 \\
\text { (SVR) }\end{array}$ & $\begin{array}{c}\text { courbe } \\
\text { de tarage }\end{array}$ & 1,29 & $<1 \%$ & $\begin{array}{c}\text { Mesure } \\
\text { topographique } \\
\text { (station totale) }\end{array}$ & $\begin{array}{l}0,86 \text { valeur } \\
\text { par défaut }\end{array}$ \\
\hline $\begin{array}{l}\text { Le Ratier à } \\
\text { Saint-Genis-les-Ollières } \\
(69, \text { Irstea) }\end{array}$ & $\begin{array}{c}1,51 \\
(\mathrm{SVR})\end{array}$ & $\begin{array}{c}\text { courbe } \\
\text { de tarage }\end{array}$ & 1,42 & $+6 \%$ & $\begin{array}{c}\text { Mesure } \\
\text { topographique } \\
\text { (station totale) }\end{array}$ & $\begin{array}{l}0,86 \text { valeur } \\
\text { par défaut }\end{array}$ \\
\hline $\begin{array}{l}\text { La Volane à } \\
\text { Vals-les-Bains } \\
(07, \text { SPC GD) }\end{array}$ & $\begin{array}{c}7,85 \\
(\mathrm{SVR})\end{array}$ & $\begin{array}{c}\text { courbe } \\
\text { de tarage }\end{array}$ & 8,25 & $-4,8 \%$ & $\begin{array}{c}\text { Mesure } \\
\text { topographique } \\
\text { (station totale) }\end{array}$ & $\begin{array}{l}0,86 \text { valeur } \\
\text { par défaut }\end{array}$ \\
\hline $\begin{array}{l}\text { La Bourbre à Jameyzieu } \\
\text { (01, DREAL RA) }\end{array}$ & $\begin{array}{c}22,8 \\
\text { (SVR) }\end{array}$ & $\begin{array}{c}\text { courbe } \\
\text { de tarage }\end{array}$ & 20,1 & $+13 \%$ & $\begin{array}{c}\text { Mesure } \\
\text { topographique } \\
\text { (station totale) }\end{array}$ & $\begin{array}{l}0,86 \text { valeur } \\
\text { par défaut }\end{array}$ \\
\hline $\begin{array}{l}\text { La Volane } \\
\text { à Vals-les-Bains } \\
(07, \text { SPC GD) }\end{array}$ & $\begin{array}{c}24,6 \\
\text { (SVR) }\end{array}$ & $\begin{array}{l}\text { courbe } \\
\text { de tarage }\end{array}$ & 24,9 & $-1,3 \%$ & $\begin{array}{c}\text { Mesure } \\
\text { topographique } \\
\text { (station totale) }\end{array}$ & $\begin{array}{l}0,86 \text { valeur } \\
\text { par défaut }\end{array}$ \\
\hline $\begin{array}{l}\text { L'Arc à St Avre } \\
(73 \text {, Irstea) }\end{array}$ & $\begin{array}{c}20,1 \\
\text { (Kalesto) }\end{array}$ & $\begin{array}{c}\text { courbe } \\
\text { de tarage }\end{array}$ & 22,1 & $-8,8 \%$ & $\begin{array}{c}\text { Mesure } \\
\text { topographique } \\
\text { (station totale) }\end{array}$ & $\begin{array}{c}0,76 \text { calculé } \\
\text { (profils } \\
\text { de vitesse au } \\
\text { camion jaugeur) }\end{array}$ \\
\hline $\begin{array}{l}\text { L'Arc à St Avre } \\
\text { (73, Irstea) LSPIV }\end{array}$ & $\begin{array}{c}97,3 \\
\text { (Kalesto) }\end{array}$ & $\begin{array}{c}\text { courbe } \\
\text { de tarage }\end{array}$ & 95,8 & $+1 \%$ & $\begin{array}{l}\text { Section mesurée par } \\
\text { camion jaugeur }\end{array}$ & 0,76 (idem) \\
\hline $\begin{array}{l}\text { L'Ardèche au Pont } \\
\text { d'Ucel (07, SPC GD) } \\
\text { Courbe de tarage }\end{array}$ & $\begin{array}{c}98,8 \\
(\mathrm{SVR})\end{array}$ & $\begin{array}{l}\text { courbe } \\
\text { de tarage }\end{array}$ & 97,0 & $+1,9 \%$ & $\begin{array}{c}\text { Mesure } \\
\text { topographique } \\
\text { (station totale) }\end{array}$ & $\begin{array}{l}0,86 \text { valeur } \\
\text { par défaut }\end{array}$ \\
\hline $\begin{array}{l}\text { L'Arc à St Avre } \\
\text { (73, Irstea) }\end{array}$ & $\begin{array}{c}111 \\
\text { (Kalesto) }\end{array}$ & $\begin{array}{l}\text { camion } \\
\text { jaugeur }\end{array}$ & 118 & $-6,3 \%$ & $\begin{array}{l}\text { Section mesurée par } \\
\text { camion jaugeur }\end{array}$ & $\begin{array}{c}0,76 \text { calculé } \\
\text { (profils } \\
\text { de vitesse au } \\
\text { camion jaugeur) }\end{array}$ \\
\hline $\begin{array}{l}\text { Le Rhône à Surjoux } \\
\text { (74, DREAL RA) }\end{array}$ & $\begin{array}{c}284 \\
\text { (SVR) }\end{array}$ & ADCP & 320 & $-11 \%$ & Suivi de fond ADCP & $\begin{array}{l}0,86 \text { valeur } \\
\text { par défaut }\end{array}$ \\
\hline $\begin{array}{l}\text { La Saône à Lyon } \\
(69, \text { CNR })\end{array}$ & $\begin{array}{c}1670 \\
\text { (SVR) }\end{array}$ & $\mathrm{ADCP}$ & 1760 & $-4,7 \%$ & Suivi de fond ADCP & $\begin{array}{c}0,90 \text { calculé } \\
\text { (profils de } \\
\text { vitesse ADCP) }\end{array}$ \\
\hline
\end{tabular}

jaugeur. Dans plusieurs autres cas les valeurs de débit mesurées au radar sont comparées aux courbes de tarage des stations hydrométriques proches. La figure 6 présente la courbe de tarage du Ratier à Saint-Genis-les-Ollières, issue de la loi d'ouvrage théorique de son seuil de contrôle à double sensibilité, et les jaugeages disponibles pour cette station.

Trois résultats du tableau 2 s'écartent de plus de $10 \%$ de la valeur dite de référence : le jaugeage de la Bourbre à Jameyzieu $(+13 \%)$ effectué dans des conditions pluvieuses, radar tenu à la main (sans support), ce qui en fait un jau- geage réalisé en situation dégradée. Le cas du jaugeage du Rhône à Surjoux (-11\%) est particulier, car le champ de vitesse utilisé est issu de la moyenne des mesures de tous les radars testés pendant la comparaison inter-laboratoire. Les dispersions observées sur les séries de vitesses enregistrées nous laissent penser que l'écho du signal renvoyé par la surface n'était pas suffisant. La figure 5 illustre les écarts à la mesure de référence en fonction du débit, on peut y voir une légère tendance à la surestimation des petits débits $\left(<2 \mathrm{~m}^{3} / \mathrm{s}\right)$ et à la sous-estimation des forts débits $\left(>2 \mathrm{~m}^{3} / \mathrm{s}\right)$. Cette tendance sera à vérifier avec d'autres mesures. 


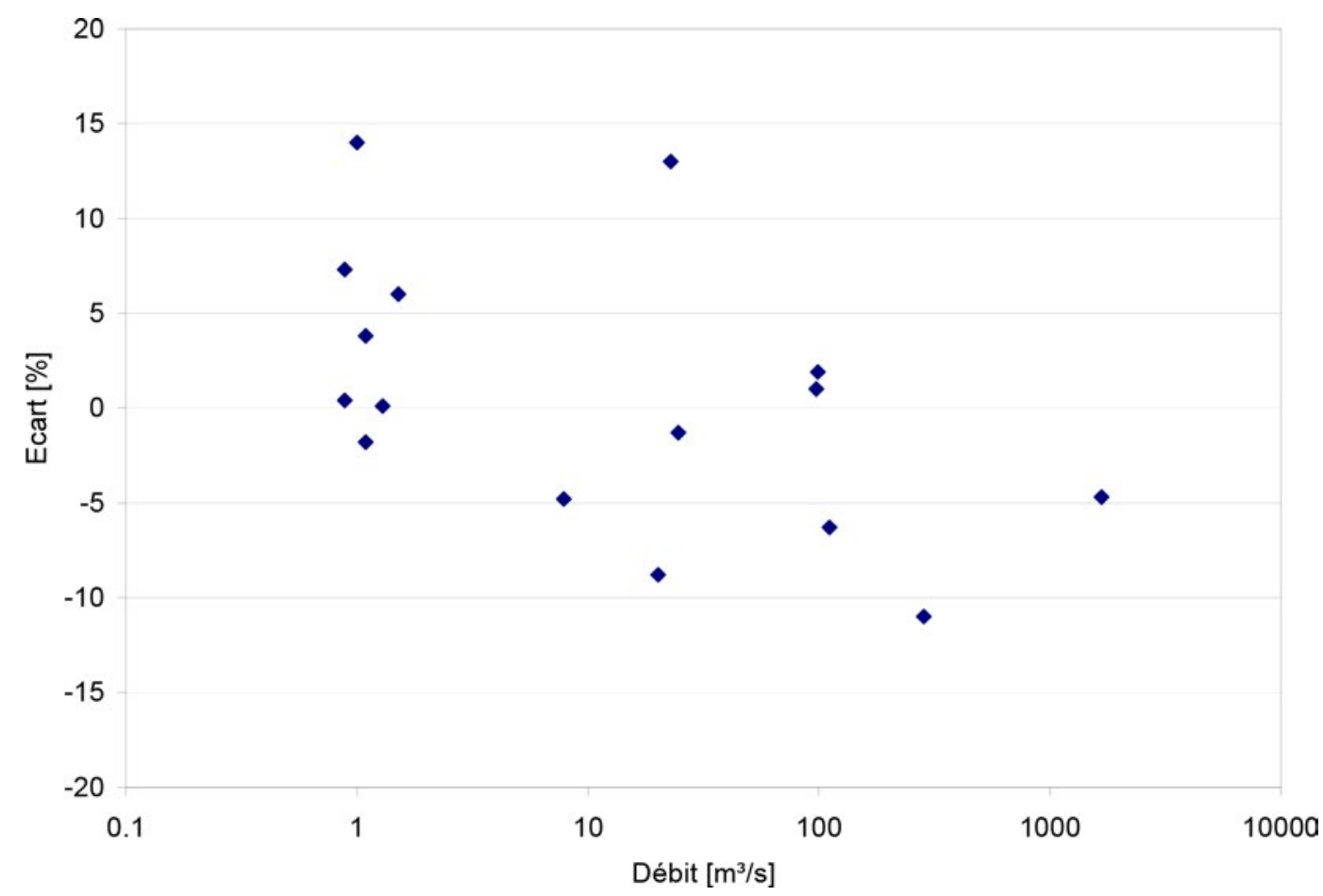

Figure 5 : Ecarts en pourcentage des débits mesurés par radar aux mesures dites de référence.

Le Ratier à Saint-Genis-les-Ollières

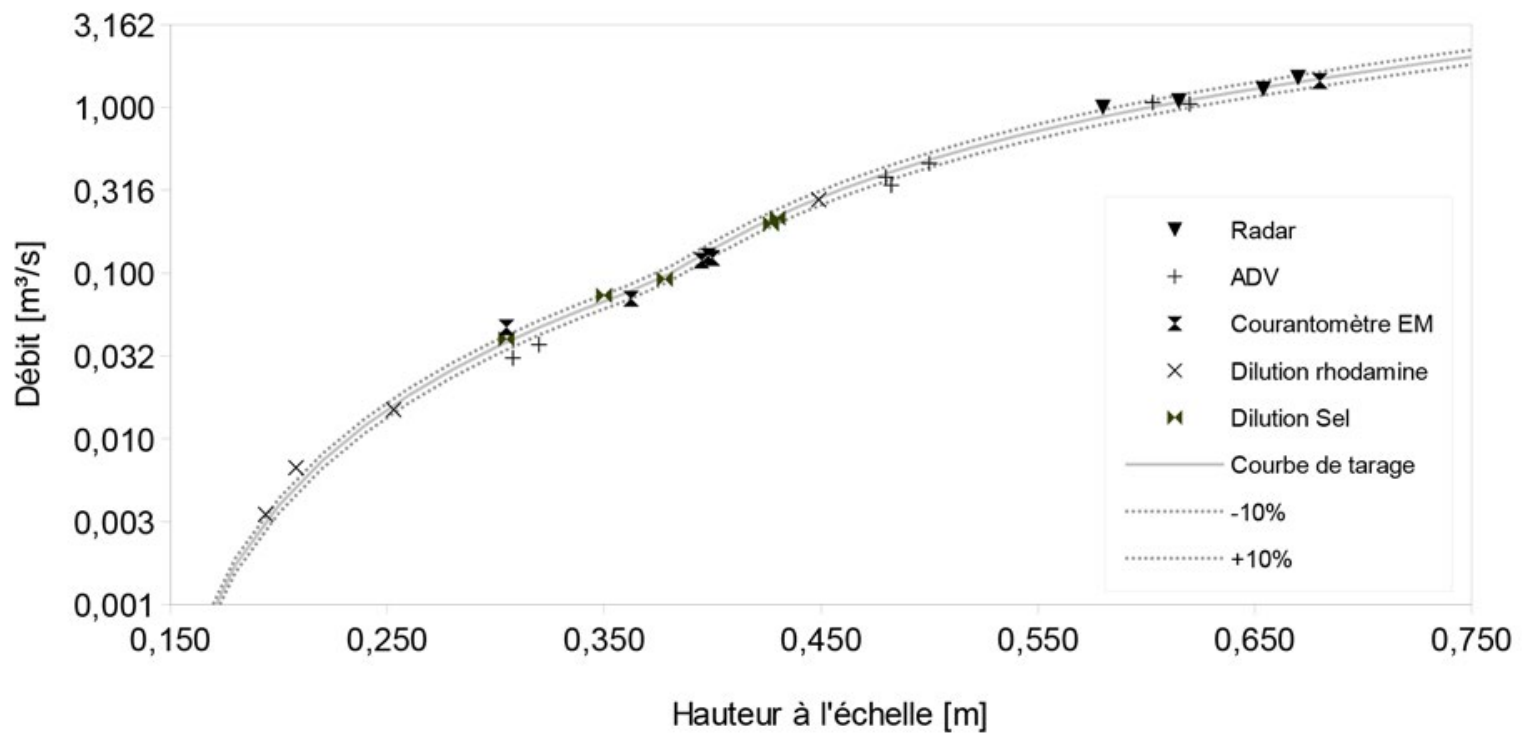

Figure 6 : Jaugeages et courbe de tarage théorique sur le Ratier à Saint-Genis-Les-Ollières (station Irstea).

La figure 6 montre la courbe de tarage et les points de jaugeages de la station hydrométrique du Ratier à Saint-Genis-les-Ollières. Cette petite rivière est suivie par Irstea et jaugée régulièrement dans le cadre du suivi de l'observatoire péri-urbain du bassin de l'Yzeron (site OTHU). La courbe de tarage a été calculée avec une formule hydraulique représentant le déversoir triangulaire et le seuil horizontal épais qui contrôlent l'écoulement (la submersion du seuil est visible vers $38 \mathrm{~cm}$ à l'échelle). Une passerelle permet des jaugeages par radar au droit de la station. Les 4 jaugeages réalisés pendant des crues de 2012 sont alignés dans les $10 \%$ autour de la courbe $(-1.8 \%,+6 \%$ et $0 \%)$ sauf le plus faible qui s'écarte de $+14 \%$. Ce cas d'application avec une courbe de tarage bien contrôlée et bien jaugée permet de valider la technique radar et d'en illustrer l'intérêt pour l'amélioration des gammes hautes eaux des courbes de tarage.

\section{CONCLUSIONS}

En définitive la technique par radar mobile offre une nouvelle solution alternative pour la mesure des débits des cours 
d'eau en crue. Cette méthode sans contact est simple à utiliser, rapide et permet des mesures dans de bonnes conditions de sécurité même pour les crues violentes. Les écarts aux valeurs de référence pour les cas traités vont jusqu'à $+14 \%$, mais sont majoritairement en dessous de $10 \%$ d'écart. La gamme de débit testée est assez large, de moins d' $1 \mathrm{~m}^{3} / \mathrm{s}$ à plus de $1600 \mathrm{~m}^{3} / \mathrm{s}$. Les recommandations présentées dans ce travail peuvent permettre aux équipes d'hydrométrie de garder un regard critique sur cette technique qu'il faut continuer à tester et améliorer pour bien en saisir les limites. La correction de l'angle d'inclinaison et la dispersion des vitesses mesurées sont les points principaux à contrôler. La surface libre ne doit pas être trop lisse pour permettre une bonne rétrodiffusion du signal. La mesure de la bathymétrie doit également être rigoureuse, répétée régulièrement sur les sections jaugées et raccordée aux échelles limnimétriques.

\section{REMERCIEMENTS}

Les auteurs remercient l'équipe métrologie d'HHLY, Marie Courtel, Fabien Thollet et les jaugeurs occasionnels du projet FloodScale sur la modélisation multi-échelle des crues cévenoles, à savoir Jessica Huza, Isabelle Braud, Marko Adamovic, Kerstin Ebert. Le projet FloodScale est soutenu par l'Agence Nationale de la Recherche (ANR) contrat $\mathrm{n}^{\circ}$ ANR 2011 BS56 027 qui contribue au programme HyMeX.

Gilles Pierrefeu, Thierry Pantel et l'équipe de la Jauge à la CNR pour leur aide sur la Saône. Patrick Duby et Paul Cluzel de l'équipe d'hydrométrie de la DREAL Rhône-Alpes pour leur disponibilité. Et enfin Guillaume Fourquet et l'équipe d'hydrométrie du SPC Grand Delta pour la collaboration en Ardèche.

\section{REFERENCES}

Bechon P.M. (2012), - Guide de l'utilisateur de BAREME, 100 p. pierre-marie.bechon@developpement-durable.gouv.fr
Costa, J., Cheng, R., Haeni, F., Melcher, N., Spicer, K., Hayes, E., Plant, W., Hayes, K., Teague, C., Barrick, D. (2006), - Use of radars to monitor stream discharge by non-contact methods. Water Resources Research, 42 (7), 0043-1397.

Dramais, G., Le Coz, J., Camenen, B., Hauet, A. (2011), Advantages of a mobile LSPIV method for measuring flood discharges and improving stage-discharge curves, Journal of Hydro-Environmental Research, 5, 301-312.

Dramais, G., Le Coz, J., Camenen, B., Hauet, A. Duby, P. (2011). Utilisation des mesures sans contact pour quantifier le débit des rivières torrentielles. Colloque SHF : " L'eau en montagne : mieux observer pour mieux prévoir », Lyon, 16-17 mars 2011. $11 \mathrm{p}$

Esteves, M., Malinur, F., (2012), Estimation du débit à partir de la mesure de vitesses de surface. Communication personnelle, 2012.

Fulton, J., Ostrowski, J. (2008) Measuring real-time streamflow using emerging technologies: Radar, hydroacoustics, and the probability concept, Journal of Hydrology, 357, 0022-1694.

Hauet, A., Kruger, A., Krajewski, W. F., Bradley, A., Muste, M., Creutin, J.-D., (2008), - Experimental system for real-time discharge estimation using an image-based method. Journal of Hydrological Engineering 13 (2), 105-110.

NF EN ISO 748 (2009), - Mesure de débit des liquides dans les canaux découverts - Méthodes d'exploration du champ des vitesses, Norme ISO, $50 \mathrm{p}$.

Le Coz, J., Hauet, A., Dramais, G., Pierrefeu, G. (2010), Performance of image-based velocimetry (LSPIV) applied to flash-flood discharge measurements in Mediterranean rivers. Journal of Hydrology. 394 (1-2) : 42-52.

Le Coz, J., Pierrefeu, G., Brochot, J.F., et al (2007), Jaugeage des rivières par ADcp: pour une culture commune, La Houille Blanche - Revue internationale de l'eau, 4, 111-118.

Le Coz, J., Pierrefeu, G., Paquier, A. (2008), Evaluation of river discharges monitored by a fixed side-looking Doppler profiler, Water Resour. Res., 44, 0043-1397.

Zolezzi, G., Zamler, D., Laronne, JB., Salvaro, M., Piazza, F., Le Coz, J., Welber, M., Dramais, G. (2011), Systematic test of surface velocity radar (SVR) to improve flood discharge prediction. Poster session, AGU, San Francisco, USA, guido. zolezzi@ing.unitn.it 